\title{
II. Bertrandit von Iveland im südlichen Norwegen.
}

\author{
Von \\ Thorolf Vogt in Kristiania.
}

(Mit. 4 Textfiguren.)

Auf einer Excursion im Frühjahr 1909 nach Sätersdalen im südlichen Norwegen besuchte ich einige Tage die noch wenig untersuchten, aber sehr mineralreichen Granitpegmatitgänge bei Iveland. Es gelang mir hier das seltene und für Skandinavien neue Mineral Bertrandit zu finden. Der Bertrandit ist von meinem verehrten Lehrer Professor W. C. Brögger in seiner Monographie über »Die Mineralien der südnorwegischen Granitpegmatitgänge $I$ «1) unter denjenigen Mineralien erwähnt, welche man auf diesen Gängen erwarten konnte, und es war mir auch deshalb eine Befriedigung, dieses Mineral identificieren zu können.

Die Pegmatitgänge werden auf Feldspat abgebaut und sind dadurch schön aufgeschlossen. Folgende Mineralien sind aus den Vorkommen in Sätersdalen von Brögger erwähnt: Beryll, Topas, Apatit, Monazit, Xenotim, Orthit, Gadolinit, Zirkon, Ilmenorutil, Columbit, Euxenit, Polykras, Blomstrandin, Fergusonit, Samarskit, Thoruranin, Thorit usw.

Der Bertrandit kommt bei Iveland als pseudomorphe Bildung nach Beryll vor. Diese Genesis ist eine für Bertrandit gewöhnliche und wird von den meisten Vorkommen dieses Minerals berichtet. Von den fran-. zösischen Fundstellen bei la Villeder ${ }^{2}$ ), Orvault ${ }^{3}$ ) und Limoges ${ }^{4}$ ), von Pisek ${ }^{5}$ ) in Böhmen wie auch vom Mt. Antero in Colorado ${ }^{6}$ ) wird die secundäre Entstehung nach Beryll von den Verff. dargetan.

1) Videnskabs-Selskabets Skrifter, math.-naturw. Klasse 1906, Nr. 6. Diese Zeitschrift 45, 82.

2) Lacroix, Bull. soc. Min. 1889, 12, 514. Diese Zeitschr. 1892, 20, 281.

3) Lacroix und Baret, Bull. soc. Min. 1889, 12, 527. Diese Zeitschr. 1892, 20, 282.

4) Michel, Bull. soc. Min. 1891, 14, 76. Diese Zeitschr. 1894, 22, 280.

5) Scharizer, diese Zeitschr. 1888, 14, 33. - Vrba, diese Zeitschr. 1895, 24, 112.

6) Penfield, Amer. Journ. Sc. 1890, 40, 488. Diese Zeitschr. 1892, 20, 492. 
Die Pseudomorphosen bei Iveland bilden hexagonale Prismen bis $1 \mathrm{dcm}$ im Querschnitt, und bestehen gewöhnlich aus einer ziemlich einförmigen, compacten Mischung von Kaliglimmer, Bertrandit und Quarz. Die Größe der einzelnen Mineralkörner wechseln innerhalb weiter Grenzen; die Bertranditkörner verschwinden bisweilen für das Auge in der makroskopisch dichten Masse, können aber andererseits bis zu einer Größe von ein Paar Centimetern heranwachsen.

Außer dem Bertrandit wurde in der Pseudomorphose Phenakit, secundärer Beryll, Muscovit, Chlorit, Orthoklas und Quarz gefunden.

Untersuchungen über diese Mineralien wie auch über einige Beryllpseudomorphosen sollen später als Beitrag zu einer gemeinschaftlichen Publication über die Mineralien der südnorwegischen Granitpegmatitgänge veröffentlicht werden. Hier wird deshalb nur der Bertrandit berücksichtigt.

Gute Krystalle von Bertrandit habe ich nur einmal in einer Beryllpseudomorphose aus dem Feldspatbruch Tveit bei Iveland gefunden. Die Hohlräume der Pseudomorphose wurden hier von zahlreichen winzig kleinen Bertranditkrystallen ausgekleidet, welche nur die Größe von 1,2 mm $\ngtr$ $0,4 \mathrm{~mm} \times 0,1 \mathrm{~mm}$ erreichten; gewöhnlich waren sie noch viel kleiner. Die Krystalle sind tafelförmig nach der Basis und nach der $a$-Axe gestreckt; eine Riefung der Flächen verläuft derselben Axe parallel.

Die Krystalle waren wegen subparallelen Zusammenwachsens wenig zu Krystallmessungen geeignet, und ich mußte deshalb eine größere Anzahl

Fig. 1.

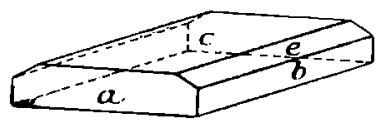

Fig. 2.

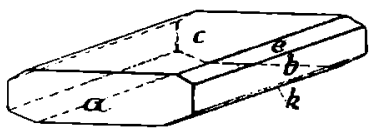

der Krystalle durchmessen, bevor einige Krystalle gefunden wurden, die befriedigenden Resultate ergaben. Die Krystalle sind von den Formen $e\{001\}$, $b\{010\}, a\{100\}, e\{011\}, k\{023\}^{*}$ und $\eta\{021\}$ begrenzt. Folgende Winkel wurden gemessen:

$\begin{array}{lllll} & n & k & \text { Gefunden: } & \text { Berechnet: } \\ b: e & 3 & 4 & 59^{\circ} 18^{\prime} & 59^{\circ} 9^{\prime} \\ b: k^{*} & 1 & 2 & 6823 & 6817 \\ b: \eta & 2 & 2 & 3949 & 3956\end{array}$

Bei der Berechnung der Winkel ist das Axenverhältnis 0,5688 : $1: 0,5973$, welches Penfield aus den Krystallen von Stoneham in Maine ${ }^{1}$ ) ermittelt

1) Amer. Journ. of Sc. 1889, 37; 213. Diese Zeitschr. 1894, 19, 79. 
hat, zugrunde gelegt. Die neue Form $k\{023\}$ trilt an einem Krystall mit zwei Flächen auf, und lieferte zwei gut übereinstimmende Messungen. Wie alle Flächen in der Zone $c: b$ war auch $k$ mit Streifung parallel der $a$-Axe versehen. Die subparallele Verwachsung nach der $a$-Axe der meisten Krystalle begründet die Streifung in der erwähnten Richtung. Die $a$-Fläche war immer sehr glatt und glänzend, war aber infolge der subparallelen Verwachsungen nicht vollkommen eben, sondern schwach convex gekrümmt. Die Krystalle sind nach der Richtung der c-Axe immer hemimorph ausgebildet, entweder mit verschiedenen Domenflächen auf der Ober- und der Unterseite, oder auch mit Domen nur auf der einen Seite. In Dünnschliffen zeigte sich dieser hemimorphe Charakter in zufälligen Schnitten parallel der $a$-Fläche, in Fällen, wo die Krystalle idiomorph gegen Quarz oder Orthoklas ausgebildet waren.

Die ziemlich unvollkommenen Krystallmessungen, wie auch die optischen Daten, deuten alle auf ein rhombisches Krystallsystem, wie es Vrba ${ }^{1}$ ) und Penfield ${ }^{2}$ ) im Gegensatz zu Scharizer ${ }^{3}$ ) behaupten.

Die bekannten Zwillingsbildungen nach $e\{011\}$ wurden mehrmals gefunden, die Individuen waren aber winzig klein, etwa 0,2-0,3 mm groß.

Fig. 3.

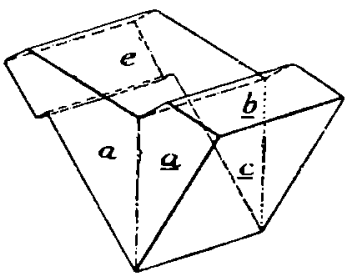

Fig. 4.

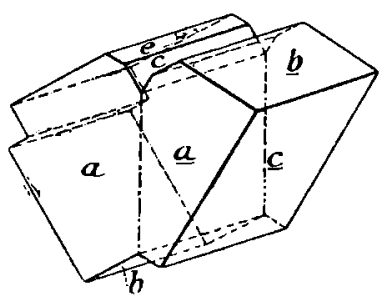

Der Winkel zwischen der Basis der zwei Individuen wurde als Mittel von zwei Bestimmungen zu $118^{\circ} 51 \frac{1}{2}^{\prime}$ bestimmt; der berechnete Wert ist 118018'. Diese Zwillinge stimmen im Habitus vollkommen überein mit denjenigen Zwillingen, welche Vrba4) aus Pisek in Böhmen bescbrieben hat.

Die rectangulären, einfachen Tafeln des Bertrandits aus Iveland ähneln im Habitus dem von Lacroix ${ }^{5}$ ) beschriebenen Vorkommen bei la Villeder in Morbihan, aber besonders dem rectangulären Typus aus Pisek in Böhmen, Fig. 5 und 6 auf der Tafel von Vrba (l. c.).

1) Diese Zeitschr. 1889, 15, 194.

2) Amer. Journ. of Science 1888, 36, 52. Diese Zeitschr, 1889, 15, 334.

3) Diese Zeitschr. 1888, 14, 33.

4) Diese Zeitschr. 1895, 24, 112.

5) Bull. soc. Min. 1889, 12, 514. Diese Zeitschr. 1892, 20, 281. 
Die physikalischen Eigenschaften des Bertrandits konnten an etwas größeren Spaltstücken (bis $1 \mathrm{~cm}$ ) studiert werden.

Von Spaltbarkeiten sind mehrere bekannt; Penfield $\left.{ }^{1}\right)$ findet Spaltung nach $m\{110\}$ und $\left.c\{001\}, V^{2} a^{2}\right)$ nach $m\{110\}$ und $b\{010\}$, und icb habe alle drei Spaltungen gefunden.

Die Spaltflächen $c$ und $b$ besitzen Perlmutterglanz, diejenigen nach $m$ Glasglanz.

Gestoßenes Bertranditpulver ist hauptsächlich aus dünnen Blättchen parallel der Basis zusammengesetzt, und diese zeolithähnliche Spaltbarkeit nach $c$ wird deshalb als die beste angesehen. Nach Wertigkeit geordnet gibt es also bei Bertrandit die drei Spaltungen $c\{001\}, b\{010\}$ und $m\{110\}$.

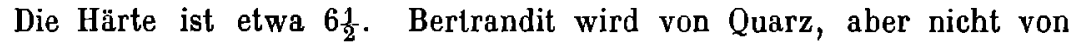
Feldspat geritzt, und ritzt selbst Feldspat. Die guten Spaltungen und die Sprödigkeit erschwerten die Bestimmung.

Das spec. Gewicht wurde in Thoulet'scher Lösung zu 2,597 bei $20^{\circ}$ ermittelt.

Bezüglich der optischen Orientierung von Bertrandit konnte ich die älteren Angaben bestätigen. Die stumpfe Bisectrix steht senkrecht auf der Basis und die Ebene der optischen Axen ist parallel $b\{010\}$.

Nach der einschlägigen Literatur scheint die optische Untersuchung des Bertrandits nicht abgeschlossen zu sein, und da mir ein sehr frisches Material in genügend großen Stücken vorlag, beschloß ich, dasselbe einer optischen Untersuchung zu unterwerfen.

In betreff der drei Brechungsindices liegt nur eine alte und approximative Angabe über den Wert von $\beta$ vor, und es war deshalb zuerst erwünscht, die drei Brechungsindices zu ermitteln.

Mittels zweier Prismen mit den brechenden Kanten parallel der $b$-Axe wurde $\beta$ nach der Methode der kleinsten Ablenkung in $\mathrm{Na}$-Licht bestimmt. Die beiden Prismen ergaben denselben Wert, nämlich $\beta_{\text {Na }}=1,6053$. Belegwinkel sind:

1.

$$
\begin{aligned}
\left.b^{3}\right) & =52040,9^{\prime} \\
\left.2 d^{4}\right) & =76 \quad 19,5 \\
\hline \beta_{N a} & =1,6053
\end{aligned}
$$

2.

$$
\begin{aligned}
b & =50^{\circ} 57^{\prime} \\
2 d & =7246 \\
\hline \beta_{N i b} & =1,6053
\end{aligned}
$$

Bertrand 5 ) gibt in seiner ersten Mitteilung über Bertrandit den approximativen Wert 1,569 für $\beta$ an. Dieser Wert ist indirect aus den Winkeln der spitzen und stumpfen Bisectrix in öl berechnet und ist nach meiner Bestimmung sehr ungenau.
1) I. c. S. 8 .
Q) 1. c. S. 8 .
3) $b=$ Prismenwinkel.
4) $2 d=$ Zweimal die kleinsle Ablenkung.
5) Bull. soc. Min. fr. 1880, 3, 96. Diese Zeitschr. 6, 293. 
Um auch die Dispersion der Lichtbrechung kennen zu lernen, wurden mittels des ersten Prismas die Brechungsexponenten für verschiedene Wellenlängen mit Hilfe eines großen Wülfing'schen Monochromators ermittelt.

\begin{tabular}{lllc} 
& \multicolumn{2}{c}{$2 d$} & $\beta$ \\
$L i$ & $75^{0}$ & $46,9^{\prime}$ & 1,6016 \\
$N a$ & 76 & 19,5 & 1,6053 \\
$T l$ & 76 & 40 & 1,6077 \\
$S r$ & 77 & 28,5 & 1,6132
\end{tabular}

Weiter wurden Prismen mit den brechenden Kanten parallel der $a$ und $c$-Axe hergestellt, um auch die Brechungsexponenten $a$ und $y z u$ bestimmen.

Die folgenden Resultate wurden dabei erhalten:

$$
\begin{aligned}
& b=34^{0} 48,5^{\prime} \\
& b=46^{0} 55,5^{\prime} \\
& \begin{array}{c}
2 d=44 \quad 5 \\
\alpha_{N^{\prime} a}=1,3914
\end{array} \\
& \begin{array}{c}
2 d=6556 \\
\gamma_{N u}=1,6126
\end{array}
\end{aligned}
$$

Das $\gamma$-Prisma war nicht ganz genau orientiert und der $\gamma$-Wert deshalb etwas zu niedrig ermittelt.

Um eine Controlle der Lichtbrechungsexponenten zu erhalten, wurde die Lichtbrechung von kleinen Spaltblättchen nach $c$ und $b$ unter Anwendung von Schroeder van der Kolk's Methode in Verbindung mit der Becke'schen Lichtlinie mittels Immersionsflüssigkeiten bestimmt, indem Mischungen von Benzol und Monobromnaphtalin in Anwendung kamen. Folgende Werte sind Mittel von zwei einander naheliegenden Werten.

$$
\begin{aligned}
& \alpha=1,5915 \\
& \beta=1,6055 \\
& \gamma=1,6145
\end{aligned}
$$

Die Bestimmungen wurden in $N a$-Licht ausgeführt. Während die Werte für $\alpha$ und $\beta$ mit denjenigen aus den Prismen übereinstimmen, liegt der $\gamma$-Wert nicht unerheblich höher. Dem letzteren möchte ich den Vorzug geben. Die drei Brechungsindices für Bertrandit sind demnach

$$
\begin{aligned}
& \alpha_{N a}=1,5914 \\
& \beta_{N a}=1,6053 \\
& \gamma_{N a}=1,6145
\end{aligned}
$$

Die Doppelbrechung $\gamma-\alpha$ beträgt nach diesen Bestimmungen 0,0231 in $\mathrm{Na}$-Licht. Um auch hier eine Controlle zu haben, wurde die Doppelbrechung mittels des Babinet'schen Compensators bestimmt. In einem Dünnschliff von der Pseudomorphose wurde ein sehr geeigneter Schnitt von Bertrandit senkrecht zu $\beta$ gefunden, und unmittelbar daneben ein Quarzindividuum, genau parallel der Hauptaxe, angetroffen. Hierdurch konnte man die 
ungenaue Dickenbestimmung mittels der Mikrometerschraube vermeiden. Es ergab sich der Wert 0,0237, welcher gut mit den obenstehenden übereinstimmt. Die Doppelbrechung ist für die verschiedenen Farben sehr wenig verschieden.

Ferner wurden Bestimmungen von dem Winkel der optischen Axen ausgeführt. In drei Spaltblättern parallel $\{001\}$ wurde der stumpfe Axenwinkel in Nußöl von der Lichtbrechung 1,4782 in Na-Licht gemessen, und folgende Werte gefunden:

$119020^{\prime}$

11937

Mittel $2 \frac{11947}{2 H_{0}=119036^{\prime}}$

Daraus ergibt sich $2 V_{0}=105^{0} 28^{\prime}$. Axenwinkelbestimmungen v.on Bertrandit sind von mehreren Mineralogen ausgeführt, nämlich von:

\begin{tabular}{|c|c|}
\hline Bertrand ${ }^{1}$ ) & $2 V_{0}=105^{0}$ \\
\hline Scharizer ${ }^{2}$ ) & $2 V_{0}=10831,5^{\prime}$ \\
\hline Vrba $\left.{ }^{3}\right)$ & $2 V_{o}=10911$ \\
\hline Penfield ${ }^{4}$ ) & $2 V_{o}=10842$ \\
\hline
\end{tabular}

Die drei letzteren Verff. haben den stumpfen Axenwinkel in öl oder im Schneider'schen Axenwinkelapparate gemessen und mittels der alten und ungenauen $\beta$-Bestimmung von Bertrand berechnet; die Werte sind deshalb zu hoch ausgefallen. Bertrand selbst hat sowohl den stumpfen wie den spitzen Axenwinkel in Öl gemessen, und daraus den wahren Winkel der optischen Axen ohne Benutzung von $\beta$ richtig bestimmen können. Den mittleren Lichtbrechungsexponenten $\beta$ hat dann Bertrand weiter unter Benutzung von dem Lichtbrechungsexponenten des Einbettungsöles berechnet; dieser Wert fällt in der Gleichung zur Bestimmung von Axenwinkeln weg, und man möchte die abweichenden Resultate in Betreff $\beta$ der Lichtbrechung des Öls zuschreiben.

Wenn man die Berechnung des wahren Axenwinkels auf Grund der neu ermittelten Werte durchführt, erbält man die gut übereinstimmenden Resultate:

$\begin{array}{ll}\text { Bertrand } & 2 V_{o}=105^{\circ} \\ \text { Scharizer } & 2 V_{o}=1050^{\prime} \\ \text { Vrba } & 2 V_{o}=10537 \\ \text { Penfield } & 2 V_{o}=10510 \\ \text { Vogt } & 2 V_{o}=10528 \\ \quad \text { Mittel } & 2 V_{o}=105019^{\prime} \\ \quad \text { und } & 2 V_{a}=7441\end{array}$

1) Bull. soc. Min. 1880, 3, 96. Diese Zeitschr. 6, 293.

2) Diese Zeítschr. 1888, 14, 33. $\quad$ 3) Diese Zeitschr. 1889, 15, 194.

4) Am. Journ. of Sc. 1888, 36, 52. Diese Zeitschr. 15, 334, 
Der Winkel der optischen Axen ist bei Bertrandit von verschiedenen Vorkommen somit sehr constant. Dies steht hauptsächlich damit im $\mathrm{Zu}-$ sammenhang, daß Bertrandit aus einer sehr reinen chemischen Verbindung besteht, beinabe ohne fremde constitutionelle Bestandteile.

Es gibt bisher drei Analysen von Bertrandit1), die alle gut mit der Formel $\mathrm{H}_{2} \mathrm{Be}_{4} \mathrm{Si}_{2} \mathrm{O}_{9}$ oder $2 \mathrm{Be}_{2} \mathrm{SiO}_{4}, \mathrm{H}_{2} \mathrm{O}$ übereinstimmen. In seiner Arbeit über den Bertrandit von Pisek wurde von Vrba auf die geometrische Äbnlichkeit zwischen Bertrandit $\left(2 \mathrm{Be}_{2} \mathrm{SiO}_{4} \cdot \mathrm{H}_{2} \mathrm{O}\right)$ und Kieselzinkerz $\left(\mathrm{Zn}_{2} \mathrm{SiO}_{4} \cdot \mathrm{H}_{2} \mathrm{O}\right)$ hingewiesen. In Anbetracht dieser Ähnlichkeit wie auch der Isomorphie zwischen Phenakit $\left(\mathrm{Be}_{2} \mathrm{SiO}_{4}\right)$ und Willemit $\left(\mathrm{Zn}_{2} \mathrm{SiO}_{4}\right)$, hat $\mathrm{Vrba}$ angedeutet, daß auch Bertrandit und Kieselzinkerz analog zusammengesetzt seien. Die chemische Formel des Bertrandits wäre dann $\mathrm{Be}_{2} \mathrm{SiO}_{4} \cdot \mathrm{H}_{2} \mathrm{O}$. Der niedrige Wassergehalt in den Bertranditanalysen wäre unter dieser Voraussetzung durch eine mögliche Decomposition des Analysenmaterials zu erklären.

Der Bertrandit von Iveland lieferte vollkommen wasserklares und frisches Material in verhältnismäßig reichlicher Menge, und schien deshalb zur Lösung dieser Frage beitragen zu können. Eine von mir in dem metallurgischen Laboratorium der Universität in Kristiania ausgeführte chemische Analyse ergab folgendes Resultat:

$\begin{array}{lrc} & & \text { Theoretisch }\left(2 \mathrm{Be}_{2} \mathrm{SiO}_{4} \cdot \mathrm{H}_{2} \mathrm{O}\right) \\ \mathrm{SiO}_{2} & 51,03 & 50,42 \\ \mathrm{BeO} & 41,45 & 42,02 \\ \mathrm{CaO} & 0,25 & - \\ \mathrm{H}_{2} \mathrm{O} & 7,18 & \frac{7,56}{100,00}\end{array}$

Die Analyse stimmt vollkommen mit den aus der Formel $\mathrm{H}_{2} \mathrm{Be}_{4} \mathrm{Si}_{2} \mathrm{O}_{9}$ berechneten Werten überein. Eine besondere Sorgfalt wurde auf die Wasserbestimmung gelegt.

Zwei verschiedene Portionen ergaben die Werte 7,06 und 7,29 (Mittel 7,18) $\mathrm{H}_{2} \mathrm{O}$, während die Formel $\mathrm{H}_{2} \mathrm{Be}_{2} \mathrm{SiO}_{6}$ einen Wassergehalt von 1/,06 verlangt. Das Wasser wurde erst bei Glühhitze abgegeben.

Durch diese an vollkommen frischem Material ausgeführte Analyse wurde somit die Formel $\mathrm{H}_{2} \mathrm{Be}_{4} \mathrm{Si}_{2} \mathrm{O}_{9}$ bestätigt und die Auffassung des Bertrandits als die Verbindung $\mathrm{H}_{2} \mathrm{Be}_{2} \mathrm{SiO}_{5}$ ausgeschlossen.

Die Resultate dieser Untersuchungen sind die folgenden:

1) Das erste Vorkommen von Bertrandit von Skandinavien wird beschrieben.

1) Damour, Bull. soc. fr. min. 1880, 3, 111. Diese Zeitschr. 6, 293.

Penfield l. c. S. 8.

Vrba (Preis) l. c. S. 8. 
2) Eine neue Form $k\{032\}$ wird gefunden.

3) Drei Spaltflächen nach $c, b$ und $m$ werden sicher constatiert. H. $=6 \frac{1}{2}$.

4) Die alte Bestimmung von $\beta$ wird corrigiert und $\alpha$ und $\gamma$, wie auch die Doppelbrechung $\gamma-\alpha$, ermiltelt.

$$
\begin{aligned}
\alpha_{N a} & =1,5914 \\
\beta_{N a} & =1,6053 \\
\gamma_{N a} & =1,6145 \\
(\gamma-\alpha)_{N a} & =0,0231
\end{aligned}
$$

5) Messungen der Axenwinkel werden ausgeführt und die Berechnung der älteren Axenwinkel mittels des neuen $\beta$-Wertes corrigiert. Als Mittel der Axenwinkel ergibt sich $2 V_{a}=74^{\circ} 44^{\prime}$.

6) Die chemische Formel $\mathrm{H}_{2} \mathrm{Be}_{4} \mathrm{Si}_{2} \mathrm{O}_{9}$ wird an vollkommen frischem Material bestätigt.

Die Untersuchungen wurden im mineralogischen Institut der Universität in Christiania, im mineralogisch-petrographischen Institut der Universität in Wien und im metallurgischen Institut der Universität in Christiania ausgeführt, und ich erlaube mir an dieser Stelle den Herren Institutsvorständen, Professoren W. C. Brögger, Fr. Becke und J. H. L. Vogt, meinen ehrerbieligsten Dank auszudrücken.

Kristiania, 4. Juni 1911. 